\title{
Analysis of Foot Pressure Variation with Change in Stride Length
}

\author{
Dr. Charudatta V. Shinde, M.S. MCh ( Orthopaedics ), Dr. Weijie Wang, Ph \\ D*, Dr. Rami J Abboud, Ph D,
}

\begin{abstract}
Additional Civil Surgeon District Dhule Maharashtra. India 424001.
Lecturer. Department of Orthopaedics and Trauma Surgery, TORT Centre, University of Dundee, Scotland, UK. Course Director (MCh Orth). Department of Orthopaedics and Trauma Surgery, TORT Centre, University of Dundee, Scotland, UK.

Statistician Department of Orthopaedics and Trauma Surgery, TORT Centre, University of Dundee, Scotland, $U K$.

Running title: Analysis of foot pressure variation with change in stride length.

Source of work: Tayside Orthopaedic and Rehabilitation Technology Centre, Ninewells Hospital and Medical School, Dundee DDI 9SY, Scotland, UK.
\end{abstract}

\begin{abstract}
The aim of this prospective randomized observational study was to identify and explain the relationship between stride length and foot pressure distribution during walking in normal adults. The objectives of the study were to collect the gait and pressure data for the normal adult subjects walking at different stride lengths and to examine whether the pressure distribution have been affected by the stride lengths. Total twenty five male adult subjects were invited to participate in this study. They were required to walk at three types of walk, i.e. self-determined longer, normal and shorter stride lengths. Their gait parameters and pressure data were synchronically recorded using GAITRite ${ }^{\circledR}$ and Pedar ${ }^{\circledR}$ systems respectively. The stride lengths were normalized by the subject leg length to avoid the confounding effect of leg length on the stride length.

In comparison of three types of walk, it was found that the peak pressure in the masked areas of the big toe, the second and third toes, the fourth and fifth toes, the first metatarsal, the second metatarsal, the medial heel and lateral heel are significantly different in three types of walk, but almost similar in the regions of the third metatarsal, the fourth metatarsal, the fifth metatarsal, the medial mid-foot and the lateral mid-foot. In the whole sole, the peak pressure is the highest in the walk with longer stride length and the lowest in the walk with shorter stride length, and the middle in the walk with normal stride length. The maximum mean pressure have the same distrubutions as those in the peak pressures. It was found that the speed, cadence and normalized stride lengths were significantly different among three types of walk.

Thus we conclude that stride length is directly proportional to peak pressure and maximum mean pressure in the foot. It is suggested that in a further study, walking speed should be kept in a constant when a subject walks at different stride lengths in order to reduce the effects from velocity.
\end{abstract}

\section{Introduction}

Locomotion, a characteristic of human, is the method by which a human moves from one geographic position to another. Walking is for locomotion, which is one of the important factors required in order to live independent life. The most common of all human body's movements is "gait", which, once learned in the early days of life, becomes almost subliminal. Gait is one of the most multifarious and well-integrated movements involving a number of joints, muscles and ligaments. The gait cycle is a continuum and this term is applied to normal gait ${ }^{1}$. Depending upon their work of pattern, every healthy individual takes an average 3500 to 8500 steps per day.

A complete gait cycle is the time interval between two consecutive events of the same foot during normal walking. This is generally recorded from the instant the foot first touches the ground until that same foot touches the ground again ${ }^{2}$. Each stride symbolize one gait cycle that is divided into two periods: stance and swing. Stance constitutes $60 \%$ of the gait cycle in which the foot remains in contact with surface. Swing constitutes the remaining $40 \%$ of the gait cycle where the foot is not in contact with surface.

During the stance period, the weight of the body imposes a pressure onto the plantar tissues of the supporting foot. The extent of this plantar pressure is determined by the strength of the loading force over the contact area of the foot ${ }^{3}$. The forces are namely mass of the body, gravity and ground reaction force which acts on the foot. Various devices, which are based on different technologies, can be used to record these variable forces on the foot. The two devices which have been used in the current study are the Pedar ${ }^{\circledR}$ and GAITRite ${ }^{\circledR}$ systems. Many studies on different pathological gait and foot disorders are done using these devices. The aim of 
the study was to recognize and describe the role of stride length in foot pressure distribution during walking in normal adults. The result of this study will enable us to identify the normal range of foot pressure values in healthy adults and its variations to stride length, if any. This will contribute to an ongoing research project on human gait evaluation and improvements in the gait training rehabilitation programme.

The aim of the study was to evaluate the effect of changes in the stride length on foot pressure distribution. The two systems, the GAITRite ${ }^{\circledR}$ gait analyser and Pedar ${ }^{\circledR}$ pressure system were used in this study. No previous study which carried out similar research to this was found, although we have done an extensive search in the literature.

\section{Objectives}

The objectives of the study were to collect the gait and pressure data for the normal adult subjects walking at different stride lengths and to examine whether the pressure distribution have been affected by the stride lengths.

\section{Materials And Methods}

Twenty five volunteers participated in this study and were recruited. All subjects were male. This study took place in the institute of Motion Analysis and Research (IMAR) of the Tayside Orthopaedics Rehabilitation and Technology (TORT) Centre, Ninewells Hospital, Dundee between October 2006 and April 2007. Ethical approval for this project was obtained from the Tayside Committee on Medical Research Ethics.

Each subject was asked to walk with shorter, normal and longer stride length. Three trails were recorded for each type of walk for each subject. GAITRite ${ }^{\circledR}$ and Pedar® system obtained nine data files from each subject, respectively. The Pedar in-sole pressure measurement system determines in-sole pressure distribution. It is a relatively easy piece of equipment to use. The GAITRite ${ }^{\circledR}$ system provides an automated means of measuring the spatial and temporal parameters of gait. All subjects were randomised by Latin square design. The way to record data ensured that the experiments were carried out properly. The researcher then recorded anthropometric measurements such as height, weight, body mass index, leg length and foot size of both sides separately. Pedar ${ }^{\circledR}$ equipment was then attached. Connections were checked. Pressure in-soles were calibrated as per size for subject. Each subject, following the same instruction, was then asked to walk with three different step lengths on the walkway. Walks were recorded on the GAITRite ${ }^{\circledR}$ system which was converted into tests using GAITRite software (version 3.4). The data was exported as an ASCII file which was then shifted to an SPSS spreadsheet for evaluation. The 'SOL' file generated by Pedar ${ }^{\circledR}$ system contains readings from each sensors of in sole. The predefined mask was created on this Pedar software which divided the in-sole into 12 distinct areas (Table1). Through Group editor several data files were combined and mask files to only one group called 'PAR' file was applied. This file was processed through the 'Group mask evaluation' function which gave statistically analysed pressure-related variables, in 12 divided regions in a group in a single 'LST' file. This file is then converted into Microsoft Excel file by a configuration program which was then shifted to SPSS spreadsheet for evaluation.

Table 1 Areas of the foot

\begin{tabular}{|l|l|}
\hline MASK & FOOT AREAS \\
\hline M 01 & Big toe \\
\hline M 02 & II \& III toes \\
\hline M 03 & IV \& V toes \\
\hline M 04 & I $^{\text {st }}$ metatarsal \\
\hline M 05 & II $^{\text {nd }}$ metatarsal \\
\hline M 06 & III $^{\text {rd }}$ metatarsal \\
\hline M 07 & IV $^{\text {th }}$ metatarsal \\
\hline M 08 & $\mathrm{V}^{\text {th }}$ metatarsal \\
\hline M 09 & Medial mid-foot \\
\hline M 10 & Lateral mid-foot \\
\hline M 11 & Medial heel \\
\hline M 12 & Lateral heel \\
\hline
\end{tabular}

SPSS (version 11.0) was used for all statistical analysis. Shapiro Wilks test was used to explore data for outliers and tested for normality of distribution. Repeated measures analysis of variance was carried out. Transformations were applied where necessary. Post-hoc comparisons were made using Bonferroni's correction for multiple comparisons. The value of significance level considered was $5 \%$ and the confidence level was $95 \%$. 


\section{Results}

The statistically significant difference $(\mathrm{P}<0.01)$ was found between three stride length walks (Table 2$)$. The normal pressure values in-sole were identified. The highest peak pressure in the foot was found in the region of the big toe. This was followed by the lateral and medial parts of the heel, the first metatarsal, second metatarsal and third metatarsal regions. The lowest peak pressure was found in the region of the medial part of mid-foot.

Table 2 Statically significances between the shorter, normal and longer stride length walks.

\begin{tabular}{|c|c|c|c|c||}
\hline \hline Stride length & Velocity & Cadence & \multicolumn{2}{|c|}{ NSL } \\
\hline \hline & & & Left & Right \\
\hline \hline Shorter & $68.94 \pm 25.48^{*}$ & $88.85 \pm 15.66^{*}$ & $1.03 \pm 0.26^{*}$ & $1.05 \pm 0.30^{*}$ \\
\hline Normal & $111.52 \pm 19.94^{*}$ & $101.22 \pm 11.65^{*}$ & $1.48 \pm 0.26^{*}$ & $1.46 \pm 0.26^{*}$ \\
\hline Longer & $133.81 \pm 81.21^{*}$ & $90.46 \pm 12.01^{*}$ & $1.99 \pm 0.27^{*}$ & $1.98 \pm 0.28^{*}$ \\
\hline
\end{tabular}

Notes: NSL - Normalized stride length.

$$
\text { * - } \mathrm{P}<0.01
$$

The reason for the higher pressure in the big toe could be due to the increase pressure exerted during the toe off phase of the gait cycle when the whole body weight passes through it. The instant peak pressure is more representative of the progression of pressures through the foot. The pressure shifts from the heel into the mid-foot, then progresses from the first to the fifth metatarsal regions and then finally to the toes. These results are comparable to other studies done on the foot pressures ${ }^{4}$.

The peak pressure is highest in the walk with longer stride length and lowest in the walk with shorter stride length. The peak pressure in the walk with normal stride length is in between other two types of walks (Figure 1 and 2).

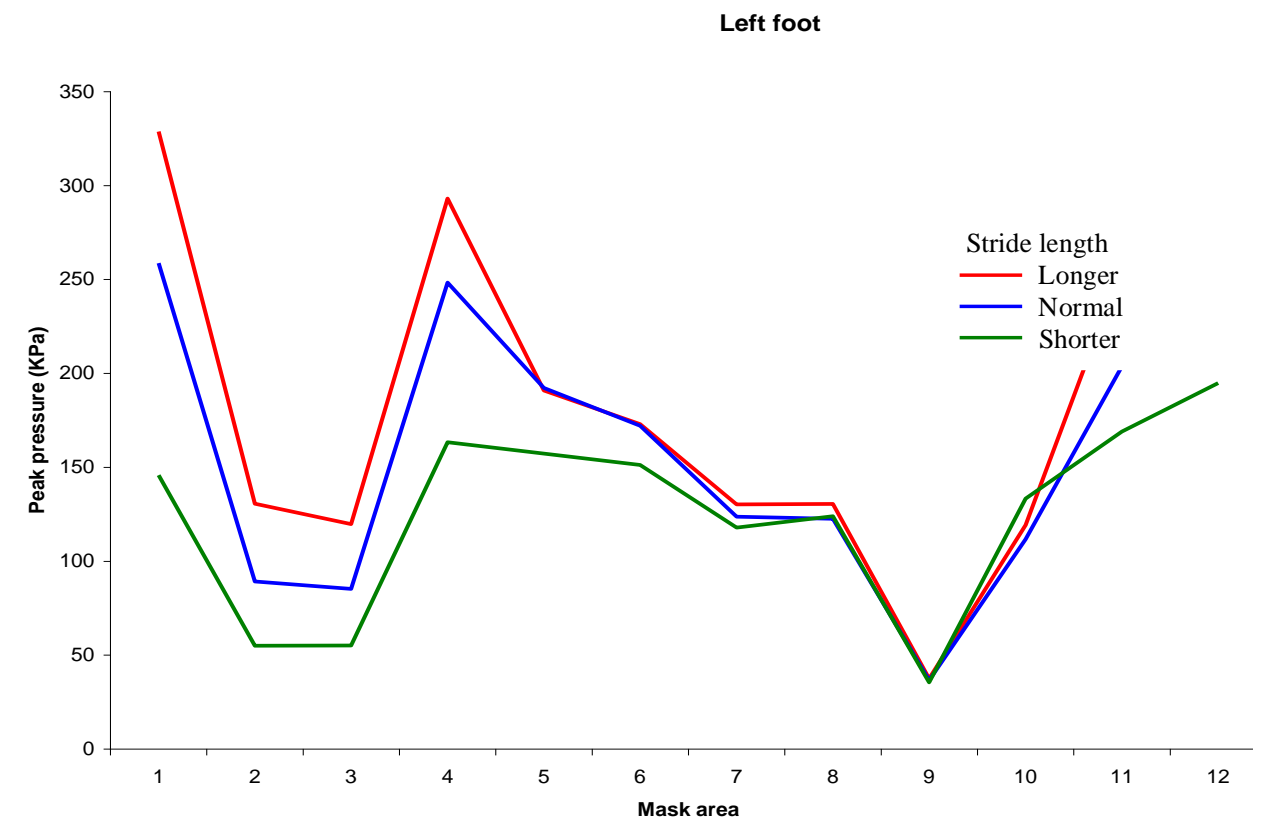

Figure 1 Peak pressure in the left foot 
Right foot

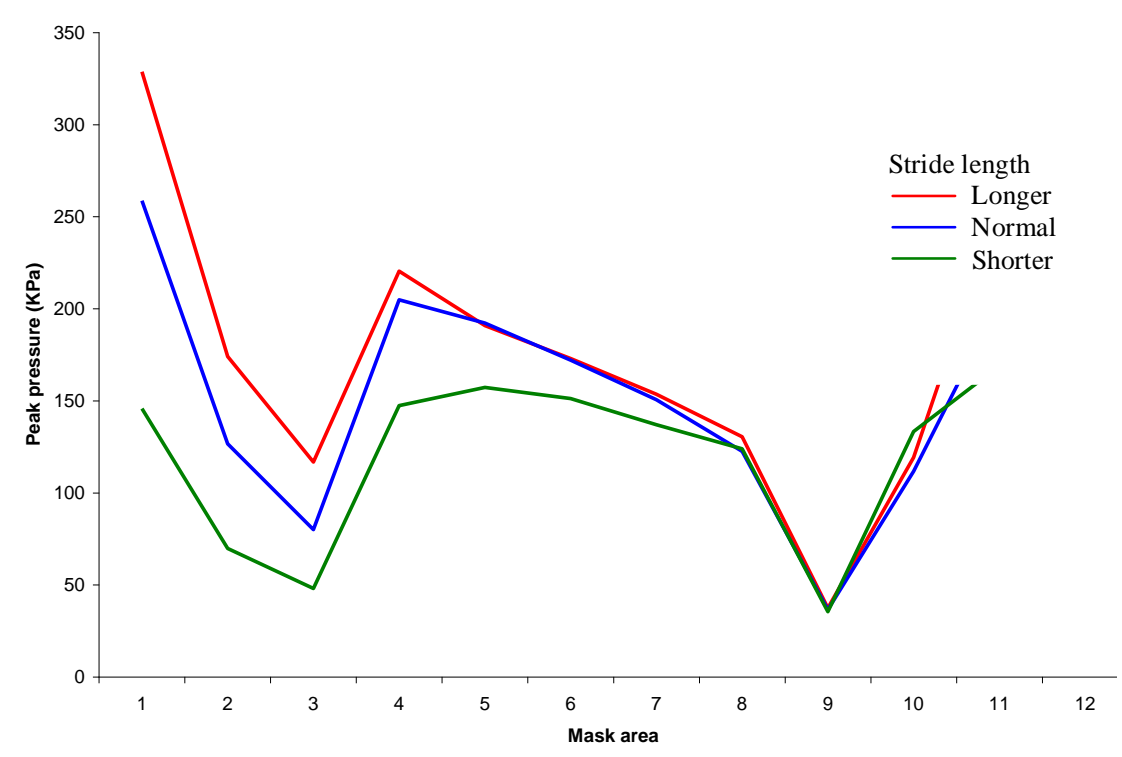

Figure 2 Peak pressure in the right foot

The maximum mean pressure is highest in the walk with longer stride length and lowest in the walk with shorter stride length. The maximum mean pressure in the walk with normal stride length is in between other two types of walks (Figure 3 and 4).

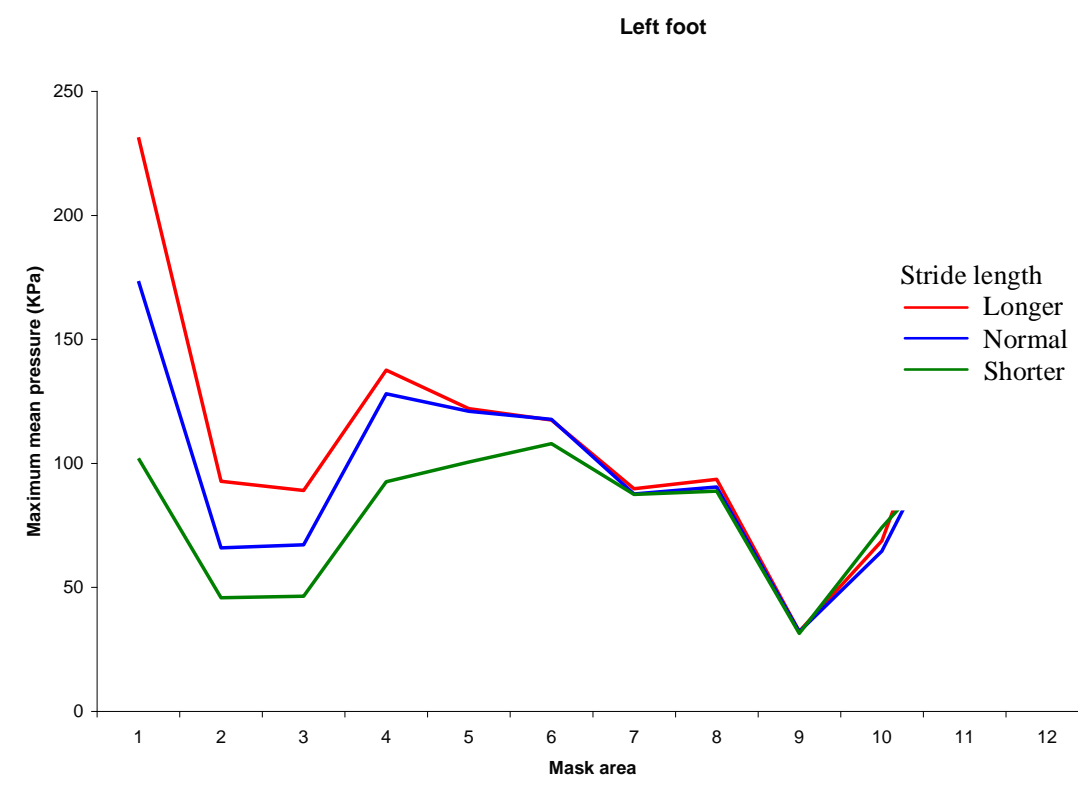

Figure 3 Maximum mean pressure in the left foot 


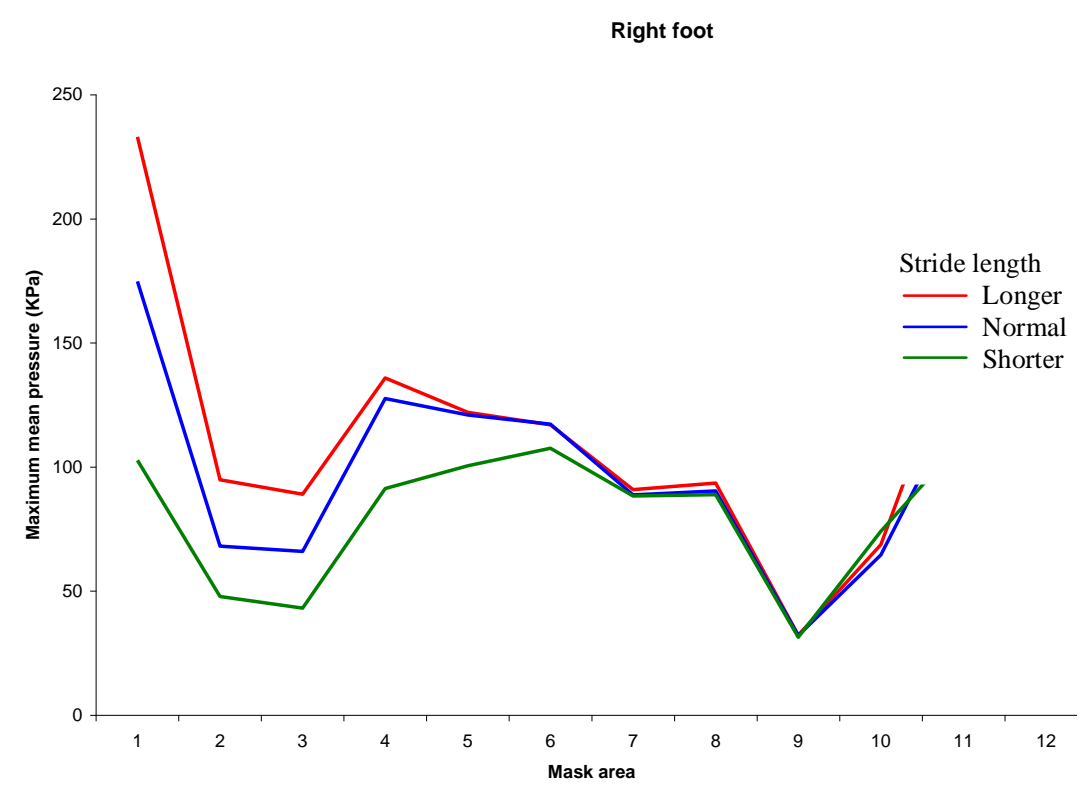

Figure 4 Maximum mean pressure in the right foot

\section{Discussion}

The mean peak pressure values in the three different types of the walk that is with shorter stride length, normal stride length and longer stride length are $248.49 \mathrm{KPa}, 310.11 \mathrm{KPa}$ and $365.95 \mathrm{KPa}$ respectively (Table $3)$.

Table 3 Peak pressure in three types of walk.

\begin{tabular}{|c|c|}
\hline Stride length & Mean peak pressure [KPa] \\
\hline Shorter & $248.49 *$ \\
\hline Normal & $310.11 *$ \\
\hline Longer & $365.95^{*}$ \\
\hline
\end{tabular}

The mean maximum mean pressure values in the three different types of the walk, that is to say, the shorter, normal and longer stride length are 109.75 KPa, 134.46 KPa and 144.47 KPa respectively. (Table 4)

Table 4 Maximum mean pressure in three types of walk.

\begin{tabular}{|c|c|}
\hline Stride length & Mean maximum mean pressure [KPa] \\
\hline Shorter & $109.75^{*}$ \\
\hline Normal & 134.46 \\
\hline Longer & 144.47 \\
\hline
\end{tabular}

* - $\mathrm{P}<0.01$

The stride length is directly proportional to peak and maximum mean pressures of the foot. The highest peak and maximum mean pressure was found in the walk with longer stride length and the lowest in the walk with shorter stride length. The peak and maximum mean pressure in the walk with normal stride length is in between other two walking ways.

The peak and maximum mean pressure in the masked areas of the big toe, the second and third toes, the fourth and fifth toes, the first metatarsal, the second metatarsal, medial heel and lateral heel are significantly different in three types of walk, but almost similar in the region of the third metatarsal, the fourth metatarsal, the fifth metatarsal, the medial mid-foot and the lateral mid-foot.

As medial mid-foot is the region with the least weight bearing area of the normal foot there is no significant difference of the presure in three types of walk. The regions like the big toe, $I^{\text {st }}$ metatarsal, medial heel and lateral heel are well known as the weight bearing areas showing siginificant rise in peak pressure with increase in stride length. 
The stride lengths were normalized by taking the ratio of the stride length to the leg length of the same side of the subject to avoid the confounding effect of leg length on the stride length. This was done to ensure the fair testing and to avoid bias.

The mean velocities for the shorter, normal and longer stride length walks were $68.94,111.52$ and $133.81(\mathrm{~cm} / \mathrm{s})$ respectively and statistically different. The standard deviation for velocities in the shorter, normal and longer stride length walks were $25.48,19.94$ and $18.21(\mathrm{~cm} / \mathrm{s})$ respectively. The ranges of velocities for the shorter, normal and longer stride length walking ways were $29.80-138.50,67.10-162.70$ and $92.20-186.70$ $(\mathrm{cm} / \mathrm{s})$ respectively (Table 5).

Table 5 The velocities in the shorter, normal and longer stride length walks.

\begin{tabular}{|l||c|c|c|c||}
\hline \multicolumn{1}{|c||}{} & \multicolumn{4}{c||}{ Velocity } \\
\cline { 2 - 5 } & Mean & Standard deviation & Minimum & Maximum \\
\hline \hline \multirow{2}{*}{$\begin{array}{l}\text { Shorter stride length } \\
\text { Normal stride length } \\
\text { Longer stride length }\end{array}$} & 68.94 & 25.48 & 29.80 & 138.50 \\
\cline { 2 - 5 } & 111.52 & 19.94 & 67.10 & 162.70 \\
\cline { 2 - 5 } & 133.81 & 18.21 & 92.20 & 186.70 \\
\hline
\end{tabular}

As there was a wide range of differences observed between the velocities in each type of walk, we think that more stable results would have been obtained if walking velocities had been controlled. It is recommended that a further study should be carried out, that is to say, a walking speed should be kept in a constant when a subject walks at different stride lengths.

* Details of the results are available on request.

\section{Acknowledgements}

It is my privilege to affirm my sincere gratitude to Ms. Kathleen McMillan (Academic communication) and Mr. Graham Arnold who was always there to solve the technical problems throughout the course of this research study.

I thank all the member of the staff in the department for their constant support.

\section{REFERENCES}

[1]. Whittle,M.W. (2002) Gait analysis: An introduction, Butterworth Heinemann, Oxford, 0-750652-62-4

[2]. Gage, J.R. (1990) An overview of normal walking. Instr Course Lect; 39:291-303.

[3]. 3) Perry, J. (1992) Gait Analysis: Normal and pathological function, SLACK incorporated, Thorofare, N.J, 1-556421-92-3.

[4]. Putti AB, Arnold GP, Cochrane L, Abboud RJ. (2007) The Pedar in-shoe system:repeatability and normal pressure values. Gait posture 25(3):401-5.

Table Legends:

Table 1 Areas of the foot

Table 2 Statically significances between the shorter, normal and longer stride length walks.

Table 3 Peak pressure in three types of walk.

Table 4 Maximum mean pressure in three types of walk.

Table 5 The velocities in the shorter, normal and longer stride length walks.

\section{Figure Legends:}

Figure 1 Peak pressure in the left foot.

Figure 2 Peak pressure in the right foot.

Figure 3 Maximum mean pressure in the left foot.

Figure 4 Maximum mean pressure in the right foot. 